\title{
DRS ASSISTING UMPIRE USING COMPUTER VISION
}

\author{
Ms. Kajal Shirke, Ms. Varsha Warise, Ms. Pooja Waykule \\ Student, Information Technology Engineering, Bharati Vidyapeeth College of Engineering, \\ Navi Mumbai,Maharashtra, India
}

\author{
Prof. S.N. Mhatre \\ Assistant Professor, Dept. of Information Technology, Bharati Vidyapeeth College of Engineering, \\ Navi Mumbai, Maharashtra, India
}

\begin{abstract}
A fair decision is crucial in any of the game to give justice to the game. Any wrong decision due to human misperception may fate the result of the game. Computer vision and Image processing techniques have been mentioned in the literature review which used multiple cameras for demonstration. This paper focuses on a system which helps in making the decisions to assist the umpire in taking the decisions such as no-ball, LBW i.e. Leg before wicket, Run out, stump out, etc with the help of smartphone camera of good quality.

The Decision review system (DRS) aims to give decisions like run-out and stump-out. Tkinter is used to develop the GUI of DRS. Object classification and object recognition is implemented using Histogram of Gradients (HOG) and Support Vector Machine (SVM). To detect the cricket ball from the video we optimized and used frame subtraction, contour detection and minimum enclosing circle algorithms using OpenCV library. Linear regression and quadratic regression are used to track and predict the motion of the ball from video source. VPython is used for the visual representation.

Section I
\end{abstract}

\section{INTRODUCTION}

\subsection{Background:}

[1] In every cricket match, umpires are responsible for deciding the approval of a ball bowled by a bowler. There are many scenarios when a delivery is disapproved by umpires. Decisions like LBW, no ball, run out and stump out requires some minutes in certain cases using television replay. So umpires make their decisions on their perception, but human perception cannot be accurate all the time. Besides, it is not always possible to conclude the accurate judgment because of the limitations of existing technology and this creates mass confusion and debates among the viewers and cricket lovers. The on-field umpire at the bowler's end signals a square mime of a TV screen to prompt the third umpire to review the decision. The third umpire initially checks if it's a legal delivery before proceeding to watch the replays to make an lbw or other decisions.

\subsection{Aim and Objective:}

It is necessary to eradicate the debate to increase the acceptability of the decisions. If this can be achieved with the help of technology, then the game will be more fair and enjoyable. Previously proposed researches regarding this field have tried to solve the problem, but these are infeasible, costly and prone to error because of the usage of sensors on field and bowlers. On the other hand, we have proposed computer vision based approach in this paper which does not need extra infrastructure because it will get video feed from broadcasting cameras. Our proposed method is expected to perform better and low cost in operating due to no infield sensor and other devices.

The remaining portion of the paper is organized as Section II includes brief picture about the Related Work and Proposed system. Section III includes Working methodology and Implementation. Section IV includes Results which discuss briefly about the the decisions got from using the various computer vision algorithms. Finally, Section V includes conclusion and future scope that lay down the outcomes of proposed work.

\section{Section II}

\subsection{Related work}

\section{LITERATURE SURVEY}

In the past few technologies was developed, one of the prominent ones being "Application of Computer Vision in Cricket: Foot Overstep No-ball Detection" proposed by AZM Ehtesham Chowdhury, Md Shamsur Rahim, Md Asif Ur Rahman [2] where image subtraction is used and this system works on only the bowler's front foot and bowling end popping crease.

"Cricket umpire assistance and ball tracking system using a single smartphone camera" by Udit Arora, Sohit Verma, Sarthak Sahni, and Tushar Sharma [3] proposed a system that involved computer vision algorithms to detect, identify and track the cricket ball and machine learning techniques to optimize and further predict various results and decisions.

In another research paper, B.L.Velammal, P. Anandha Kumar proposed "An Efficient Ball Detection Framework for Cricket" [4]. In that non-ball objects and ball objects are identified. Region Growing segmentation is chosen for segmentation. After performing segmentation, the ball and non-ball objects are differentiate based on shape properties. The non-ball objects are eliminated and resulting frames consists of only ball object. The ball objects are used further to 


\section{International Journal of Engineering Applied Sciences and Technology, 2021 Vol. 5, Issue 11, ISSN No. 2455-2143, Pages 262-266 \\ Published Online March 2021 in IJEAST (http://www.ijeast.com)}

detect the ball.

"Implementation of Augmented Reality in Cricket for Ball Tracking and Automated Decision Making for No ball" by Nikhil Batra, Harsh Gupta, Nakul Yadav, Anshika Gupta, Amita Yadav [5] proposed a system that determines whether a ball is a no-ball or wide ball. The system applies different techniques like canny edge detector, Hough line transform algorithms, Douglas Peucker algorithm contour.

No More Third Umpires" by Alex Joseph, Alistar Fernandez, Jasir Ahamed P.A., Treesa Joseph [6] proposed a system that uses image and video processing techniques, which automates the role of the third umpire.

\subsection{Problem statement}

In India, everyone is enthusiastic about cricket. Many training academies, competitions, and even though gully cricket restricts the use of decision review system for making a decision because of its high cost. Because of that many unfair decision was given by the umpire which is not good for both the teams, as a result, many controversies have happened. Our project aims to develop a computer system that helps the umpire for making a decision in cricket at a low cost and can be used at various competitions, academies and even in gully cricket.

\section{PROPOSED SYSTEM}

Our project "DRS assisting umpire using computer vision" is implemented using Python and Tkinter. GUI of our project i.e. DRS gives options to choose from to the user which contains Stump out, Run out, No ball and LBW decisions. Stump out and Run out can be decided by playing the source video in slow or fast motion. Further decisions like LBW and No Ball can be implemented by extracting frames through the source video. Ball detection and Batsman height detection is done from the extracted frames. The detected ball coordinates are further used for ball tracking. 3D coordinates are mapped into 3D coordinates and smoothen using regression techniques. Visualization is implemented through Vpython which shows results for all these decisions.

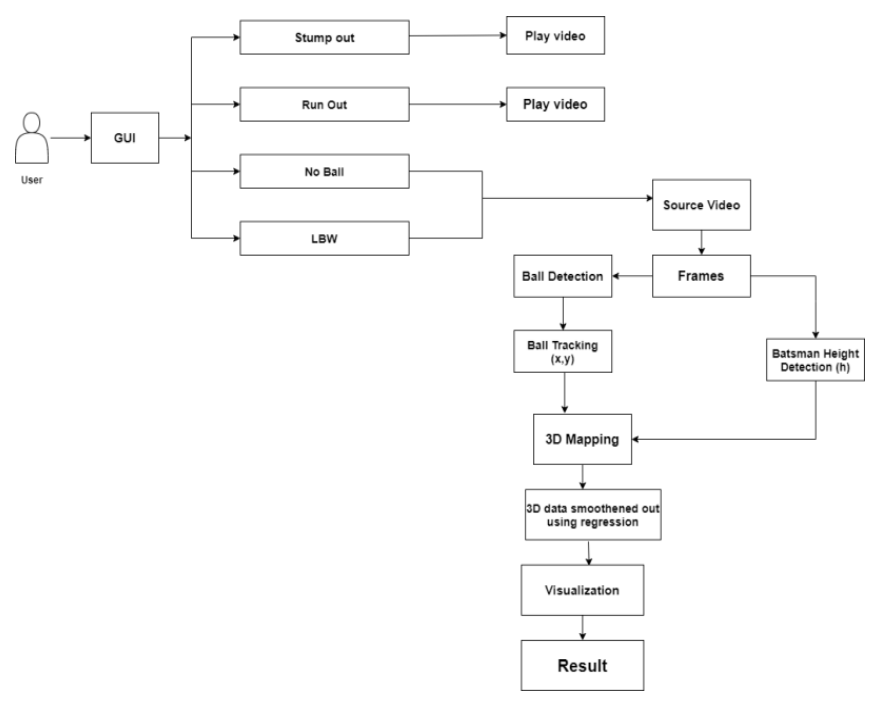

Figure 3:Proposed System

\subsection{Ball Detection}

Frame differencing technique is used to extract frames which further converted into grayscale. These frames are then used extract HOG features which then fed to SVM model which is built using OpenCV SVM library is then used to detect the ball by using frame subtraction method. Positive and negative data samples are collected which are also used to extract HOG features.

\subsection{Ball Tracking}

SVM model is used to detect the exact presence of the ball where the ball is detected in the particular frame. A separate file is created to store the coordinates of the ball which are obtains as ' $x$ ' and ' $y$ ' coordinates.

\subsection{Batsman Detection and Tracking}

OpenCV 's built-in People Detector SVM models are used to detect the batsman from the provided video. The batsman movement is tracked by limiting the search window to the neighborhood of the first detection which is displayed on the frame.

\subsection{Mapping 2D coordinates to 3D world coordinates}

The 2D coordinates of the tracked ball which are obtained after series of transformation using Minimum Enclosing Circle algorithm are then mapped into 3D world coordinates according to the cricket pitch dimensions. The z-coordinates i.e. depth is obtained by comparing it with the radius which is obtained at the beginning vs. Obtained at the end of the pitch. It is then scaled the ratio to the length of the pitch.

\subsection{D Visualization of Tracked ball and Cricket Pitch}

The obtained $\mathrm{x}, \mathrm{y}$ and $\mathrm{z}$ coordinates are used to visualize the objects in 3D using VPython visualization library. For better visualization, linear and quadratic regression is applied over various dimensions. 


\section{International Journal of Engineering Applied Sciences and Technology, 2021 \\ Vol. 5, Issue 11, ISSN No. 2455-2143, Pages 262-266 \\ Published Online March 2021 in IJEAST (http://www.ijeast.com)}

\section{Section III}

\section{WORKING METHODOLOGY}

We developed a system that helps the third umpire to make a decision on various calls on the field.

\subsection{DRS Gui}

It Provides facilities to give any decision like No-ball, Stumpout, LBW and Run out. Tkinter is used to make GUI of the Decision review system and various computer vision algorithms are used to give these decisions based on criteria.

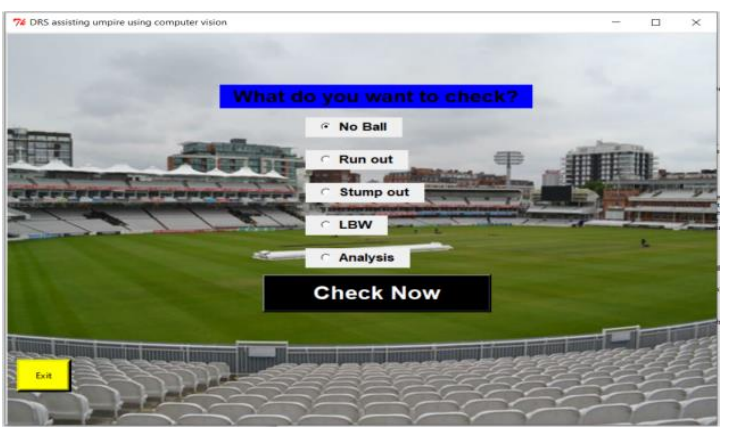

Figure 4.1:DRS Gui

\subsection{Stump Out}

The Stump-out decision is determined by playing the video in slow motion also in fast motion based on further conditions. If batsman out of his ground leaving no part of his bat or body behind the crease in an attempt to hit the ball and the wicketkeeper dislodges the bails, he/she is termed out. In case the keeper displaces the bails before the ball reaches him/her, the batsman will not be given out.

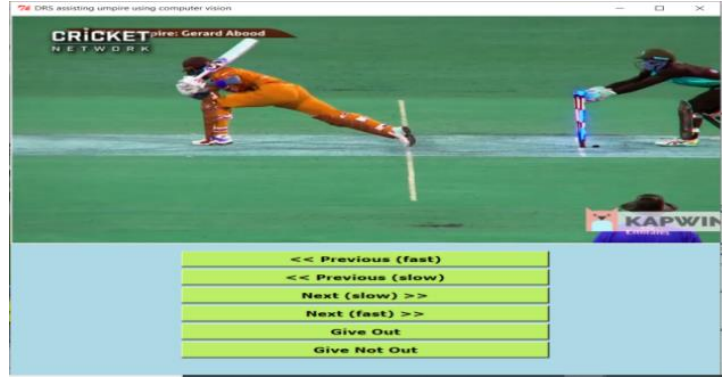

Figure 4.2:Stump out decision

\subsection{Run Out}

To determine the run-out decision the system plays the selected video in slow motion as well as in fast motion. If the wicketkeeper or any of the fielder displace the bails while either of the batsmen fails to make his ground while running between the wickets, the batsman is determined as a run-out. The batsmen must have something behind the line to be safe except they are on the same side, in that case, the batsman further away from the stumps determines as run-out. A fielder must touch the ball before it hits the wicket for the run-out to be genuine. If the batsman hits the ball and it strikes the stumps on the other end while the other side batsman or non-striker is out of the crease and the bowler has touched the ball before it hits the wicket then it will be determined as run-out.

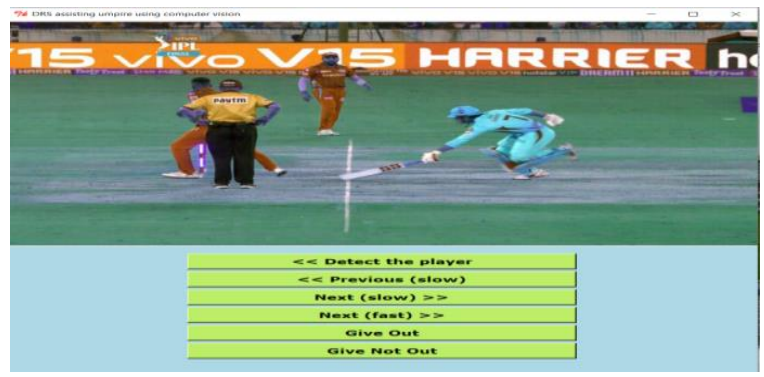

Figure4.3:Run out decision

\subsection{Leg Before Wicket (LBW)}

LBW decision is to determine in the way that if the ball hits the batsman without hitting the batsman bat or a hand holding a bat or going to hit the wicket, then he is to be adjudged out LBW, unless:

(1) the ball pitched only on the leg side or

(2) the ball hit the batsman outside the off-stump and the umpire determines that the batsman was genuinely attempting to play the ball

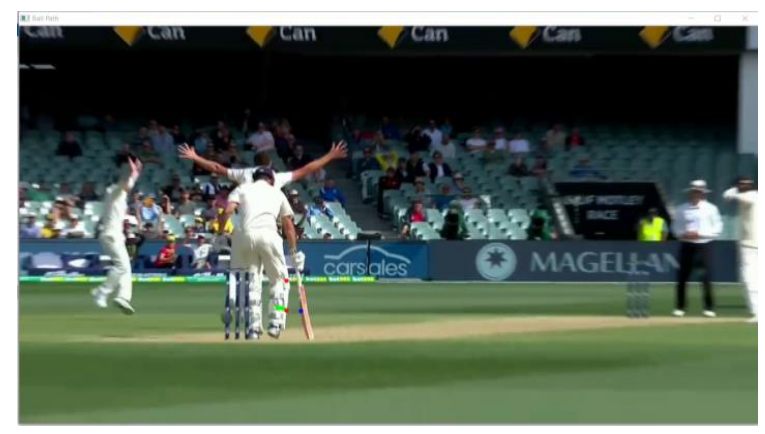

Figure 4.4:Ball Path

\subsection{No Ball}

To determine the no-ball first we have to locate the position of the ball nearest the batsman wicket crease then if the ball hasn't bounced off the ground, and the ball height is greater than the batsman height, it is determined as a no-ball. 


\section{International Journal of Engineering Applied Sciences and Technology, 2021 \\ Vol. 5, Issue 11, ISSN No. 2455-2143, Pages 262-266 \\ Published Online March 2021 in IJEAST (http://www.ijeast.com)}

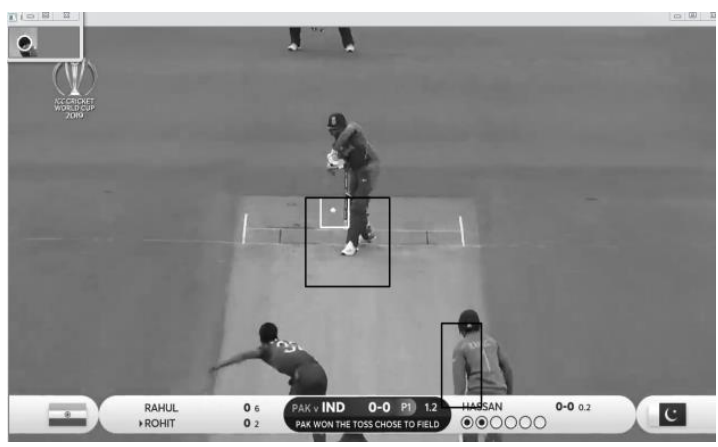

Figure 4.5(1):Searching Window

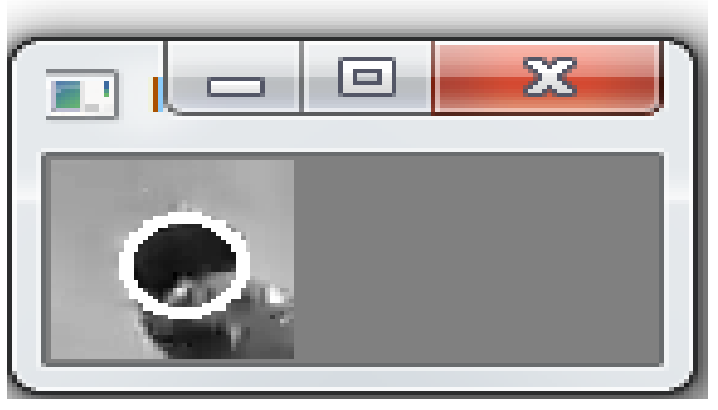

Figure 4.5(2): Best fit Circle

Section IV

\section{RESULTS}

Our project DRS assisting umpire using computer vision gives the following results:

1. Stump out and Run-out decisions by playing video either in slow or fast motion.

2. LBW decision based on

i) The impact is outside or inside.

ii) Wicket hitting the stump or not hitting the stump.

3. No ball decision by detecting

i) The position of the ball nearest the batsman wicket crease

ii) And the ball hasn't bounced off the ground, and the ball height is greater than the batsman's height.

\section{Section V}

\section{CONCLUSION}

The main objective of our project is to assist the umpire in game of cricket to make fair decision in efficient way. Our project discusses the use of computer vision algorithms to extract frames and to detect, tracked the ball from the provided source video. Vpython helps to visualize the results of various decisions which makes the UI more attractive and interactive. Also use of single smartphone camera makes the system cost efficient and easy to use.

\section{FUTURE SCOPE}

This system is designed mainly for training academies, gully crickets and cricket competitions at a small level, but can be used at an advanced level for national and international level cricket matches by including multiple cameras, microphones which may be useful for more accurate and deep analysis.

\section{REFERENCES}

[1] Chowdhury AZM, Rahim Shamsur., and Rahman Asif-Ur. (2016) "Application of computer vision in Cricket: Foot overstep no-ball detection," DOI: 10.1109/CEEICT.2016.7873086 3rd International Conference on Electrical Engineering and Information Communication Technology (ICEEICT), IEEE (pp. 1-5).

[2] Arora Udit, Verma Sohit, Sahni Sarthak and Sharma Tushar (2017) "Cricket umpire assistance and ball tracking system using a single smartphone camera" DOI:10.7287/peerj.preprints.3402 (pp. 1-14).

[3] B.L.Velammal, P. Anandha Kumar (2010) “An Efficient Ball Detection Framework for Cricket" International Journal of Computer Science Issues (IJCSI), ISSN (Online): 16940784 Vol. 7 (pp. 30-35)

[4] Batra Nikhil, Gupta Harsh, Yadav Nakul, Gupta Anshika, Yadav Amita. (2014). "Implementation of augmented reality in cricket for ball tracking and automated decision making for no ball'.DOI: 10.1109/ICACCI.2014.6968378. (pp. 316321).

[5] Joseph Alex, Fernandez Alistar, Jasir Ahamed P.A., Treesa Joseph (2018) "No More Third Umpires" International Research Journal of Engineering and Technology (IRJET), e-ISSN-2395-0056 (pp. 225-227).

[6] Dakhare Bhawana, Khatu Amish, Singh Hrushikesh, Gupta Aniket, (2020) "Visual E-commerce Application using Deep Learning" International Research Journal of Engineering and Technology (IRJET), Volume: 07 (pp. 4102-4110).

[7] Patil, V., Ingle, D.R. (2021) An association between fingerprint patterns with blood group and lifestyle based diseases: a review. Artif Intell Rev 54, 1803-1839 (2021). https://doi.org/10.1007/s10462-020-09891-w

[8] Semwal A., Mishra D., V. Raj, Sharma J., and Mittal A., (2018) "Cricket shot detection from videos," 9th International Conference on Computing, Communication and Networking Technologies (ICCCNT). IEEE, 2018, DOI:10.1109/ICCCNT.2018.8494081 (pp. 1-6).

[9] Simon Siregar, Ismail bin Ibrahim, Muhammad Ikhsan Sani, Marlindia Ike Sari (2018) "Design of Computer 
Vision Based Ball Detection System on Wheeled Robot Soccer” DOI:10.1109/ICCEREC.2018.8711988, IEEE (pp. 46-49).

[10] K. Rasool Reddy, K. Hari Priya, N. Neelima (2015) "Object Detection and Tracking - A Survey" DOI:10.1109/CICN.2015.317 (pp. 418-421).

[11] Yi-You Hou, Sz-Yu Chiou, Ming-Hung Lin (2015) "Real-time Detection and Tracking for Moving Objects Based on Computer Vision Method" IEEE DOI: 10.1109/ICCRE.2017.7935072 (pp. 213-217).

[12] Shantaiya S., and Mehta K. V., (2015) "Multiple Object Tracking using Kalman Filter and Optical Flow," European Journal of Advances in Engineering and Technology, vol. 2, 2015, (pp. 34-39).

[13] Kale K, Pawar S., and Dhulekar P., (2015) "Moving Object Tracking using Optical Flow and Motion Vector Estimation,” DOI: 10.1109/ICRITO.2015.7359323 (pp. $1-6)$.

[14] T. D’Orazio, N. Ancona, G. Cicirelli, and M. Nitti, (2002) "A ball detection algorithm for real soccer image sequences", Proceedings of ICPR, DOI: 10.1109/ICPR.2002.1044654, (pp 210-213).

[15] Vito Reno, Nicola Mosca, Roberto Marani, Massimiliano Nitti, Tiziana D'Orazio,(2018) "Convolutional Neural Networks based ball detection in tennis games", IEEE/CVF Conference on Computer Vision and Pattern Recognition Workshops DOI: 10.1109/CVPRW.2018.00228, (pp.1839-1845). 